\title{
Practical Guidance for Non-Specialist Physical Therapists Managing People with Hemophilia and Musculoskeletal Complications
}

\author{
Angela Forsyth ${ }^{*}$, Greig Blamey ${ }^{2}$, Sébastien Lobet ${ }^{3}$, Paul McLaughlin ${ }^{4}$ \\ ${ }^{1}$ REBUILD Program, Diplomat Specialty Infusion Group, Cincinnati, OH, USA \\ ${ }^{2}$ Manitoba Inherited Bleeding Disorders Program, Health Sciences Center, Winnipeg, MB, Canada \\ ${ }^{3}$ Cliniques Universitaires Saint-Luc, Service d'Hématologie, Brussels, Belgium \\ ${ }^{4}$ Hemophilia Centre and Thrombosis Unit, Royal Free London, London, UK \\ Email: *aforsyth@diplomat.is
}

How to cite this paper: Forsyth, A., Blamey, G., Lobet, S. and McLaughlin, P. (2020) Practical Guidance for Non-Specialist Physical Therapists Managing People with Hemophilia and Musculoskeletal Complications. Health, 12, 158-179.

https://doi.org/10.4236/health.2020.122014

Received: December 16, 2019

Accepted: February 14, 2020

Published: February 17, 2020

Copyright (c) 2020 by author(s) and Scientific Research Publishing Inc. This work is licensed under the Creative Commons Attribution International License (CC BY 4.0).

http://creativecommons.org/licenses/by/4.0/

\begin{abstract}
People with hemophilia (PWH) have impaired blood coagulation and hemostasis. Bleeding into joints and muscles is the main manifestation of the disorder, causing severe joint damage and muscle complications. Lifelong, regular physical therapy is therefore important for $\mathrm{PWH}$, in order to support recovery from bleeds and reduce recurrences. Ideally, these individuals should be in the care of a hemophilia treatment center (HTC), where a physical therapist (PT) should be a regular part of the comprehensive team. However, not all PWH have convenient access to an HTC, and they may be referred to local non-specialist PTs, who may have only limited experience with the condition, for day-to-day physical therapy treatment. This article, written by four experienced hemophilia PTs, introduces key aspects of the pathology of the hemophilic joint in adult PWH with musculoskeletal complications or established joint disease (or those with inhibitors) that are relevant to PTs. The characteristic differences between the joints of $\mathrm{PWH}$ and those of people with similar pathologies are clarified. Practical advice on how the physical therapy consultation can be adjusted for PWH is given, and the importance of communicating with the HTC emphasized. The aim of this narrative review is to raise awareness of hemophilia and joint disease for non-specialist PTs, and serve as helpful background information for PTs who encounter PWH with musculoskeletal complications or established joint disease in their clinics.
\end{abstract}

\section{Keywords}

Hemophilia, Joint Diseases, Physical Therapy 


\section{Introduction and Aims}

Hemophilia A and B are inherited disorders characterized by deficient or missing coagulation factors VIII or IX, respectively, of which the main long-term clinical manifestation is joint damage [1] [2]. People with hemophilia (PWH) have joint and muscle bleeding that results in arthropathy, joint destruction, pain, limited range of motion (ROM), and muscle weakness, which in turn affect functional mobility, balance, posture, and strength [2] [3] [4]. Physical therapy may be helpful to preserve or improve the joint mobility, function, and muscle strength of PWH [5] [6].

Where available, the main pharmaceutical treatment for hemophilia is clotting factor replacement therapy-the intravenous infusion of factor VIII or IX concentrate, to replace the missing or reduced coagulation factor [3]. Prophylactic treatment can be used to prevent bleeding for people with severe hemophilia; adherence to the prescribed infusion schedule is important, in order to maintain factor concentrations at clinically effective levels and avoid musculoskeletal complications [7]. However, the availability of pharmaceutical treatment is not equal worldwide. On-demand treatment, which aims to treat a bleed after symptoms have appeared, is more prevalent when resources are scarce [1] [3]. Older PWH are also more likely to have been treated on-demand historically, and are therefore more likely to have musculoskeletal complications caused by bleeding into their joints [8].

Ideally, PWH should have a link to a hemophilia treatment center (HTC), where a multidisciplinary team (typically including a hematologist, nurse, physical therapist [PT], and psychosocial professional) provides comprehensive care to address issues related to the bleeding disorder [7]. However, there is regional variability in the provision of HTCs, such that they may not be easily accessible to all PWH [9] [10]. Indeed, due to geographical, financial or other constraints, considerable numbers of PWH are not regularly cared for in an HTC [9] [10]. Therefore, some PWH who need frequent physical therapy may be more conveniently served in their local community. In these cases, PWH may be referred to providers with limited experience in hemophilia [7].

This article is the result of the authors' pooled specialist hemophilia experience totaling 77 years. It aims to provide practical advice for PTs who may be specialists in orthopedics, rheumatology or other areas, but who do not have experience treating adult $\mathrm{PWH}$ and who therefore may not be familiar with the nuances of the disorder. We focus specifically on adult PWH with musculoskeletal issues and established joint disease (hereafter simply referred to as " $\mathrm{PWH}$ "), and those patients with inhibitors. Younger individuals and those without musculoskeletal issues may have a different profile, due to availability of earlier and more consistent prophylactic treatment [8], and are therefore considered outside the scope of this review. Although many of the same principles apply when treating the general population, there are important differences and considerations in hemophilia. We discuss these aspects, describe joint pathology 
in $\mathrm{PWH}$, and provide practical guidance for managing these individuals. These are important topics for non-specialist PTs, as having an awareness of the main issues affecting joints in hemophilia builds PWH's confidence and trust that the physical therapy treatment will not cause a bleed.

\section{Musculoskeletal Bleeding and the Joint in the Adult with Hemophilia}

Musculoskeletal bleeding episodes, including hemarthrosis (joint bleeding), make up approximately $80 \%$ of all bleeds in PWH and can cause a high degree of joint damage and functional limitations if there is no rehabilitation [1] [6]. All synovial joints have the potential for hemarthrosis, and bleeding into the intraarticular joint space occurs when capillaries in the inner layer of the synovial lining leak blood into the space as a result of vessel injury [2] [11]. This injury may result from local trauma, repetitive stress, or a simple movement affecting the synovial membrane [1] [2] [11]. Even a single hemarthrosis can set a process in motion that results in joint disease later in life, but the risk of joint damage increases with each subsequent hemarthrosis [2] [11].

Acute hemarthrosis in $\mathrm{PWH}$ presents as a burning or tingling sensation in the joint, which progresses to an inflamed joint that becomes tense and warm to the touch, along with pain and a reduced ROM, as well as possible erythema of the skin (Figure 1(a)) [6] [12]. As treatment strategy is dependent on the type of hemarthrosis, and symptoms may be similar, differential diagnosis is critical.

Joint hemarthroses cause both direct and indirect damage to the synovial membrane and cartilage (Figure 2) [1]. Acute synovitis (inflammation of the synovial membrane) can become chronic, and hypertrophy of the membrane, which is generally palpable, may lead to easily triggered sub-acute hemarthroses [1] [2] [12]. These typically present with tolerable pain and minor joint immobility [12]. Joints that are affected by repeated bleeding ("target joints") should be monitored closely to avoid permanent damage [2]. Monitoring strategies used by the authors of this article include adherence to hemophilia medical treatment as well as functional and physical performance assessment, and imaging techniques such as ultrasound. Joint health can also be monitored with the Hemophilia Joint Health Score (HJHS) [13], although this is quite a complex tool that should only be used by PTs trained in its use.

Repeated hemarthroses in target joints hinder recovery of ROM and muscle strength [2]. They can also trigger multiple episodic joint swelling [6] [14], which causes changes within the joint and may be one of the key reasons for poor proprioception and joint awareness, unless the person undergoes rehabilitation at each occurrence [5] [6]. Importantly, PWH may also have sub-clinical bleeding episodes that, although not detectable by them or their PT on physical examination, can also cause joint damage over time, due to exposure of the structures to blood [15].

Blood in joints results in chondrocyte death, causing cartilage matrix degradation and permanent cartilage and bone degeneration, ultimately giving rise to 
(a)

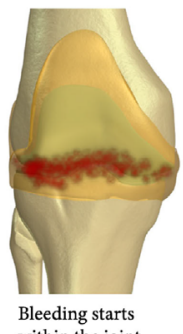

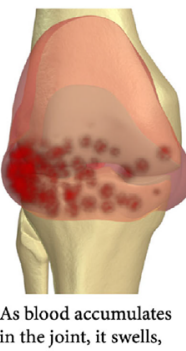

in the joint, it swells.

becomes warm to the touch and may be painful. Appropriate treatment at this stage will stop further bleeding into the joint

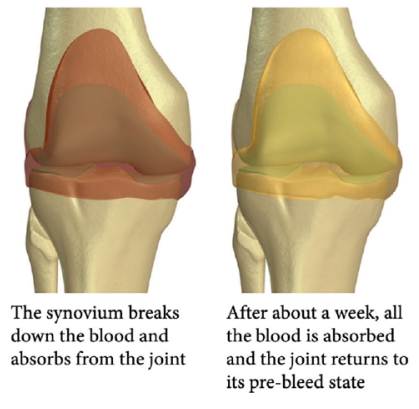

and the joint returns (b)

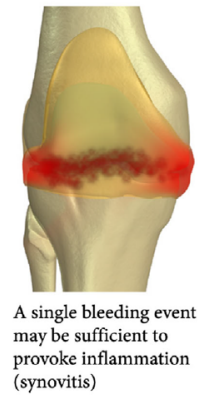

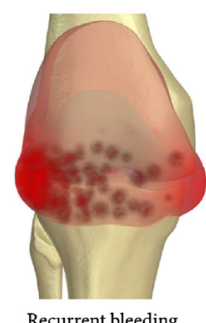

Recurrent bleeding leads to swelling of the joint and ongoing synoviti

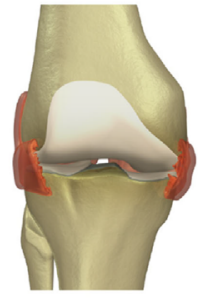

Growth of the joint lining (synovium) leads to an inflamed, vascular and fragile tissue that is mor likely to bleed. Further bleeds can
destroy the cartilage

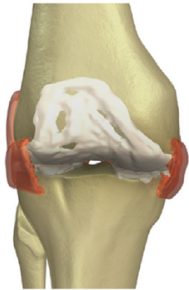

Destruction of cartilag leads to long-lasting joint damage, resulting in arthritis and stiffened joints

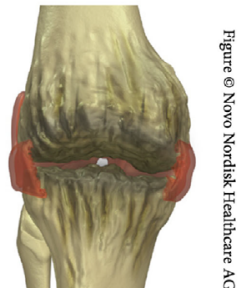

In later stages, there is complete loss of cartilage and the bone may become deformed changing the shape of the joint

Figure 1. The hemophilic joint. (a) Short-term recovery from a single hemarthrosis, and (b) Long-term damage caused by recurrent bleeding. Reprinted from TalkingJoints ${ }^{\circledR}$ [22] with permission from Novo Nordisk.

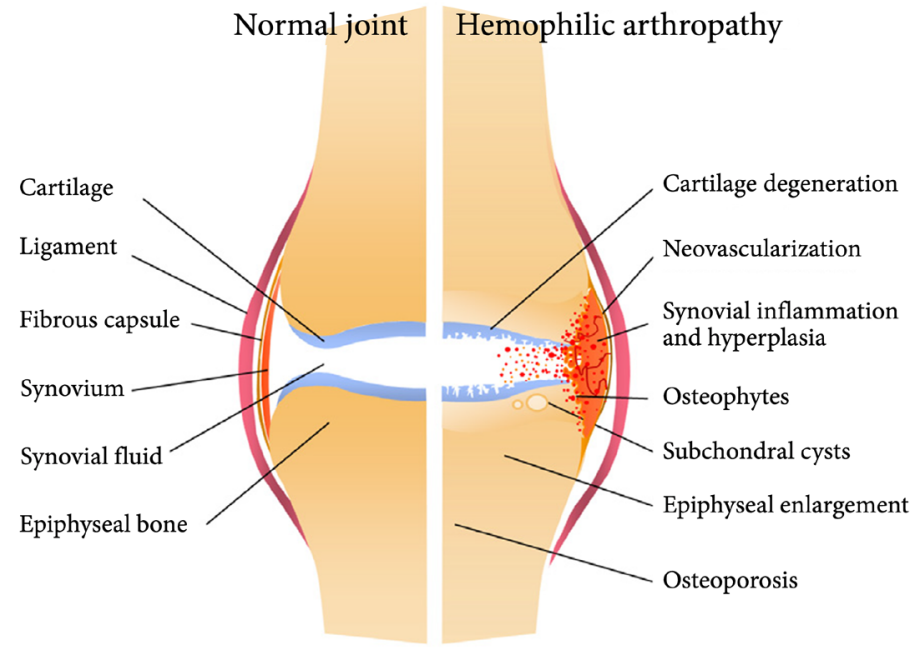

Figure 2. The articulation in hemophilia. Image credit: Pulles et al. 2017 [1]. Reprinted from Pharmacological Research, Vol 115, Pulles AE, Mastbergen SC, Schutgens RE, Lafeber FP, van Vulpen LF, Pathophysiology of hemophilic arthropathy and potential targets for therapy, 192-199, Copyright (2017), with permission from Elsevier.

hemophilic arthropathy_joint disease caused by bleeding in PWH (Figure 1(b)) [1] [11] [16] —and causing persistent pain in the joint [12]. Polyarthropathy may also develop from frequent hemarthroses in multiple joints, even with appropriate 
and adequate factor concentrate management and access to specialized hemophilia care and physical therapy. In such cases, physical therapy aims to improve joint mobilization and help prevent further soft tissue fibrosis or contracture and muscle atrophy [14].

Elective orthopedic surgery, including joint replacement, is frequently performed in PWH with established joint disease to reduce pain and disability [17]. Such surgery, which is challenging because of the extent of pre-existing joint damage [18], should be undertaken by an experienced multidisciplinary team in the HTC setting, in order to achieve optimal outcomes [7] [19]. During the post-operative period, hemostatic coverage must be ensured with an adequate clotting factor replacement regimen [7]. Physical therapy and rehabilitation is recommended post-surgery, but input from the HTC team is required [7].

In people with hemophilic arthropathy and those recovering from surgery, physical therapy intervention can address muscle strength, maintain joint mobilization and function, and play an important role in both prevention of and recovery from hemarthroses [4] [20] [21].

\section{Adults with Hemophilia and Musculoskeletal Complications}

The level of joint damage present in PWH varies worldwide, depending on the individual person's ability to receive adequate treatment or other variables that result in poorly managed bleeding. Additionally, some PWH develop antibodies against the clotting factor ("inhibitors"), and bleeding and joint disease can be more severe than usual in these patients [23] [24]. In our experience, PWH may therefore have a higher degree of joint damage than expected, and PTs who do not have experience treating PWH may not have encountered this in other practice areas. PWH may have a greater level of joint damage present at a younger age than other patients (e.g. those with rheumatoid arthritis), and frequently in multiple joints. Early cartilage damage in the joint is initiated by bleeding, with most children with severe hemophilia having their first joint bleed by 4 years of age [25]. However, function may be better than expected, and PWH can still benefit from therapeutic intervention. It is important to assess all joints and establish a baseline for their functional mobility before starting a rehabilitation program. When designing the physical therapy or rehabilitation program, an awareness of global joint involvement and a risk vs benefit analysis of all chosen assessment and intervention techniques is critical in order to avoid triggering bleeding episodes.

As a consequence of long-lasting severe joint disease, $\mathrm{PWH}$ are likely to experience a chronic, baseline level of pain in multiple joints [26] [27]. Additionally, they may have acute pain layered on top of chronic pain when there is bleeding or a flare in arthropathy symptoms [27] [28]. Baseline pain in PWH may be higher than that in the general population due to multiple arthrophic joints and advanced joint damage, but some individuals may show better acceptance of chronic pain from living with multiple affected joints [27] [29]. To assess problematic pain, PTs 
can use standardized questionnaires and scales [29] [30] [31] [32]. When taking a medication history in PWH, it is important to understand that some medications and herbal supplements can impede the coagulation process and are therefore strictly contraindicated (for example acetylsalicylic acid or St John's wort [7] [33]). PTs should be aware that some PWH may have access to pain medications prescribed for them by other practitioners, or may be taking over the counter pain medication, without input from the HTC. Therefore, PTs need to have a working knowledge of which pain medications and herbal supplements may adversely affect factor treatment or bleeding complications, and are therefore contraindicated for PWH. The HTC can advise and provide guidance to PTs on this.

Perception of pain may vary between individuals with hemophilia: often, older individuals may have become accustomed to baseline levels of pain, but pain may peak during bleeding events, especially major bleeding events [27] [29] [32]. It is important to prioritize the most troublesome pain when seeing $\mathrm{PWH}$, and to understand that it is unlikely they will become pain-free. PTs should discuss pain with PWH similarly to how they would counsel people with chronic pain in the general population: the emphasis should be on management rather than treatment, and on meeting goals that are not purely focused on reducing or eliminating pain.

Owing to musculoskeletal bleeding complications and joint damage, balance impairments, proprioception issues and reduced muscle strength may have accumulated over a lifetime [34] [35] [36]. For example, many older PWH may have had long periods of immobility or joint immobilization in their growing years [17], which likely influenced the development of their motor co-ordination strategies [35] [37]. Changes of the joint articulation surfaces may, in time, affect balance and dynamic stability [38]. Therefore, it is the authors' opinion that balance issues need to be monitored and treated regularly and carefully, as they may persist if not managed properly. PTs can provide adaptive strategies and equipment to maintain and improve function despite joint changes.

Hematomas can also affect muscle strength, and may lead to shortening of the muscle due to fibrosis [18] [21]. Maintaining adequate strength is important to provide dynamic stability to the joints to aid in the prevention of hemarthroses [21]. It is our opinion that physical therapy intervention is unlikely to be able to return strength to levels associated with people without hemophilia, as PWH may have had years of muscle atrophy and contractures. However, the PT can still work towards and encourage a state of wellness by carefully planning individualized, supervised exercise programs (which may include strength, flexibility and cardiovascular exercises), even if there is advanced joint disease or poor joint health or function [39]. Indeed, fitness and wellness benefits were observed in a study after a six-week exercise program in PWH, including in individuals with complications and co-infections [39]. However, timelines for progress and treatment protocols typically used in the general population are generally not suitable in PWH. Programs for PWH should consider the joint- and muscle-status 
of the individual, and therefore should start conservatively, be pain-free for the individual, and intensify only when previous exercises have been tolerated successfully [39]. Furthermore, the positive effects of individualized exercise on wellbeing have also been shown in other conditions that are often comorbid with hemophilia, such as HIV infection. In one such study, patients with HIV showed improvements in physical function and quality of life after a 10-week exercise and wellbeing program [40].

PWH may have adapted to their arthropathy and therefore present with features that are characteristic of hemophilia joint damage. The unique, characteristic posture of some PWH (see Table 1 and Figure 3) is caused by multiple joint involvement and soft tissue contractures that are not always possible to correct [14] [41]. Likewise, PWH may develop an unusual gait to compensate for multiple arthrophic joints, in order to achieve the most efficient way of walking [42] [43] [44] [45]. Trying to force a textbook posture, such as a neutral hip position, or gait change, such as heel strike, may be detrimental as it may place further stress on other affected joints and soft tissue and may cause bleeding complications. It may be useful to observe how PWH have adapted: how they move, their footwear, the differing heights of their crutches to accommodate

Table 1. Adaptive postures and positions of comfort for PWH following joint bleeds (and one muscle bleed).

\begin{tabular}{|c|c|c|c|}
\hline \multirow{2}{*}{ Bleeds } & \multicolumn{3}{|c|}{ Adaptive postures and position of comfort } \\
\hline & Position of comfort & Habitual posture & Potential problems \\
\hline Ankle ${ }^{a}$ & Plantarflexion & $\begin{array}{l}\text { Walking on toes, with knee } \\
\text { and/or hip flexed to compensate }\end{array}$ & $\begin{array}{l}\text { Ankle in unstable position, } \\
\text { with small area of weight-bearing } \\
\text { on talus and sole of foot; overuse } \\
\text { of calf muscles; pressure on knee }\end{array}$ \\
\hline Shoulder ${ }^{\mathrm{a}}$ & Adduction, internal rotation & Arm held close to body & $\begin{array}{l}\text { Difficulty with activities related to } \\
\text { daily living and self-care }\end{array}$ \\
\hline Wrist and fingers ${ }^{\mathrm{a}}$ & Flexion & Wrist flexed, hand closed & $\begin{array}{l}\text { Difficulty extending wrist and fingers; } \\
\text { inefficient grip }\end{array}$ \\
\hline Toes $^{\mathrm{a}}$ & Extension (dorsiflexion) & Extension (dorsiflexion) & Difficulty wearing shoes \\
\hline Hip flexor (iliopsoas) ${ }^{\mathrm{b}}$ & $\begin{array}{l}\text { Hip flexion, some external rotation } \\
\text { and increased lumbar lordosis }\end{array}$ & $\begin{array}{l}\text { Hip flexed, extreme lordosis, } \\
\text { walking on toes }\end{array}$ & $\begin{array}{l}\text { Back pain; incomplete hip extension; } \\
\text { stress on knee and ankle }\end{array}$ \\
\hline
\end{tabular}

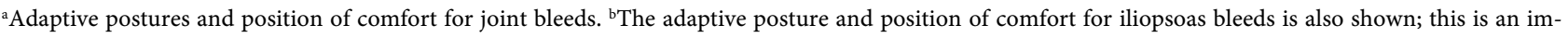
portant muscle bleed that is commonly worsened with physical therapy if proper precautions are not taken. This table does not constitute a complete list of the bleeds that may lead to movement restrictions and/or adaptive postures in PWH; several muscle bleeds have been omitted for brevity. Adapted with permission from: Table 1 in Exercises for People with Hemophilia. (c) World Federation of Hemophilia 2006. http://elearning.wfh.org/resource/exercises-for-people-with-hemophilia/ [41]. 


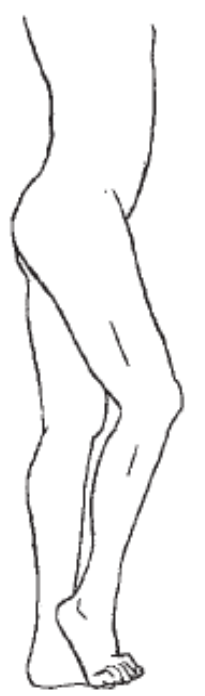

(a)

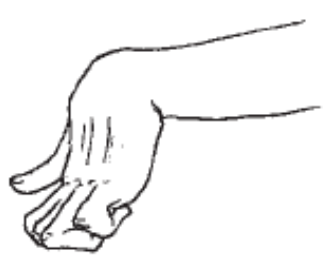

(b)

Figure 3. Flexion deformities that may be observed in PWH. (a) Hip flexion contracture caused by psoas retraction. To compensate for the hip flexion, a knee flexion contracture occurs, while an equinus compensates for the shortened leg; (b) Wrist and finger retraction caused by a Volkmann contracture, as a result of a forearm bleed. Adapted with permission from: Figure 3 in Exercises for People with Hemophilia. (C) World Federation of Hemophilia 2006. http://elearning.wfh.org/resource/exercises-for-people-with-hemophilia/ [41].

ROM restrictions in their elbows, etc. The selection of assistive devices must consider multiple joint involvement while simultaneously working toward the ultimate goal of maximizing functional mobility and independence.

Therefore, we believe that when managing $\mathrm{PWH}$ with established musculoskeletal complications, PTs should focus more on improving function and strength within the available and symptom-free ROM and on preventing progressive loss of ROM, rather than on increasing ROM. This strategy is similar to how PTs would treat and manage patients with advanced osteoarthritis (OA) before joint replacement. Additionally, supporting $\mathrm{PWH}$ and helping them adapt to their situation and develop appropriate physical compensations may be more beneficial than applying corrective strategies that attempt to achieve normal biomechanics in joints with advanced joint disease.

\section{Differences and Similarities between People with Hemophilia and Other Patients Seen by Physical Therapists}

Although some elements are similar, PWH and people with OA have a different underlying pathogenicity, which results in multiple arthrophic joints in hemophilia [46]. This leads to major differences in balance, strength and ROM, compared with patients with OA. In hemophilia, cartilage lesions are diffuse [5]

[47], while in the general population requiring physical therapy the lesions are usually more focal, especially in young people with joint damage (see Table 2) [48]. 
Table 2. Differences in joint arthropathy in PWH, or individuals with osteoarthritis or rheumatoid arthritis.

\begin{tabular}{|c|c|c|c|}
\hline & $\begin{array}{l}\text { Hemophilic arthropathy (HA) } \\
\text { [1] [2] [54] [55] }\end{array}$ & $\begin{array}{l}\text { Osteoarthritis (OA) } \\
\text { [56] [57] [58] }\end{array}$ & $\begin{array}{l}\text { Rheumatoid arthritis (RA) } \\
\text { [59] [60] [61] [62] [63] }\end{array}$ \\
\hline $\begin{array}{l}\text { Shared features } \\
\text { with HA }\end{array}$ & - & $\begin{array}{l}\text { - Cartilage degeneration and } \\
\text { osteophyte formation }\end{array}$ & $\begin{array}{l}\text { - Synovitis, cartilage degradation, } \\
\text { and bone resorption }\end{array}$ \\
\hline Presentation & $\begin{array}{l}\text { - Painful acute onset } \\
\text { - Joint swelling and damage }\end{array}$ & $\begin{array}{l}\text { - Gradual joint swelling } \\
\text { and enlargement }\end{array}$ & $\begin{array}{l}\text { - Gradual painful joint } \\
\text { swelling and damage }\end{array}$ \\
\hline Pathophysiology & $\begin{array}{l}\text { Bleeding directly from synovial } \\
\text { lining into the joint }\end{array}$ & $\begin{array}{l}\text { Mix of risk factors, including age, } \\
\text { sex, injury, wear and tear, obesity, } \\
\text { genetics } \\
\text { - } \quad \text { Biochemical processes involving } \\
\text { synovium, cartilage, and bone }\end{array}$ & $\begin{array}{l}\text { - } \text { Genetic basis } \\
\text { - } \text { Chronic autoimmune disorder } \\
\text { - } \text { May also include systemic and } \\
\text { extra-articular manifestations }\end{array}$ \\
\hline Major processes & $\begin{array}{l}\text { - Hemarthrosis-induced, } \\
\text { widespread synovial inflammation } \\
\text { Acute inflammatory mediators and } \\
\text { iron-induced oxygen metabolites } \\
\text { cause chondrocyte apoptosis and } \\
\text { hemosiderin deposition } \\
\text { - Iron increases pro-inflammatory } \\
\text { cytokines that increase pannus in } \\
\text { synovium } \\
\text { Widespread cartilage destruction } \\
\text { and bone changes }\end{array}$ & $\begin{array}{l}\text { Synovial inflammation, } \\
\text { mostly in regions adjacent to } \\
\text { cartilage and bone damage } \\
\text { Activated synovium releases } \\
\text { cytokines that accelerate } \\
\text { destruction of adjacent cartilage }\end{array}$ & $\begin{array}{l}\text { - Autoimmune-mediated } \\
\text { synovitis causes pannus } \\
\text { (through angiogenesis), } \\
\text { and leads to bone destruction } \\
\text { - Enzymes from synoviocytes } \\
\text { and chondrocytes destroy } \\
\text { cartilage }\end{array}$ \\
\hline Age of onset & $\begin{array}{l}\text { - First joint bleed usually occurs } \\
\text { in infants (age }<2 \text { years) in most } \\
\text { severe cases (varies with severity } \\
\text { and quality of prophylaxis) }\end{array}$ & $\begin{array}{l}\text { Present in } 50 \% \text { of people } \\
>65 \text { years of age with } \\
\text { chronic knee pain }\end{array}$ & $\begin{array}{l}\text { Present in } 3 \%-4 \% \text { of } \\
\text { people } 65-74 \text { years of age, } \\
\text { with prevalence of onset } \\
\text { decreasing with age }\end{array}$ \\
\hline Severity depends on: & $\begin{array}{l}\text { - Number of hemarthroses } \\
\text { - Cumulative biomechanical stresses } \\
\text { - Access to medical care, factor } \\
\text { infusion concentrate and } \\
\text { physical therapy }\end{array}$ & $\begin{array}{ll}\text { - } & \text { Age } \\
\text { - } & \text { Time since onset } \\
\text { - } & \text { Risk factors (see above) }\end{array}$ & $\begin{array}{ll}\text { - } & \text { Age at diagnosis } \\
\text { - } & \text { Access to disease-modifying } \\
\text { drugs } \\
\text { - } & \text { Smoking status } \\
\text { - } & \text { Disease duration } \\
\text { - } & \text { Joint erosions }\end{array}$ \\
\hline
\end{tabular}

Some information included in this table is based on clinical experience/observations of the authors.

Although the same structures are commonly affected both in acute injury or arthritis in the general population and in hemophilic bleeding (joint capsule, synovial membrane, proprioceptors, muscles, cartilage, bone), the mechanism of injury is different. Whereas a person without hemophilia may have acute injury and resolution of the insult, in hemophilia a bleeding component is layered on acute injury and causes lingering consequences that are worsened with repeated bleeding. For example, an individual with hemophilia with an ankle sprain will have damage to the ligaments as well as possible bleeding into the muscle or joint. For PWH with an ankle affected by multiple bleeds, balance is affected by episodic joint swellings and the resulting extended periods of immobility. In hemophilia, when the synovium is injured and bleeding occurs, muscle spasms and pain result [14] [45]. Repeated hemarthroses cause distension of the joint 
capsule, which leads to mechanoreceptor lesions [14] [45]. This contrasts with the situation in the general population, where trauma (such as a sprain) causes tearing, which then results in mechanoreceptor lesions [49]. Therefore, being able to differentiate pain caused by different pathologies is important. Differentiating between an acute joint bleed and arthropathy can be done by assessing the signs and symptoms present. This is a difficult task, and an ultrasound study has shown that even PWH and experienced hemophilia physicians can misdiagnose a painful joint (both failing to identify a bleed, or identifying a bleed incorrectly) [50]. Differentiating factors include: the type of pain; the course of symptoms and their cause; the history of the joint before presentation of current symptoms; and how impaired ROM is during the episode (see Figure 4) [51] [52] [53].

ROM tends to decrease with age in both $\mathrm{PWH}$ and healthy people, but in the former ROM limitations can begin at an early age and are associated with the number of hemarthroses already experienced [64] [65] [66]. Chronic bleeding into the joint and muscle, inflammation, cartilage damage, and arthropathy lead to limited ROM in PWH, and can be worsened if physical therapy is not conducted [43] [67] [68]. In clinical practice, it is commonly accepted that reduced ROM and muscle shortening can occur in $\mathrm{PWH}$ from prolonged protective guarding during hemarthroses (holding the joint in a position of comfort), or from joint limitations due to arthropathy. Loss of ROM may be acute-during an active bleed the joint capsule distends and motion is limited-or chronic, due to complications from previous hemarthroses or hematoma. Progressive loss of ROM significantly correlates with difficulty in walking and increased energy expenditure [44].

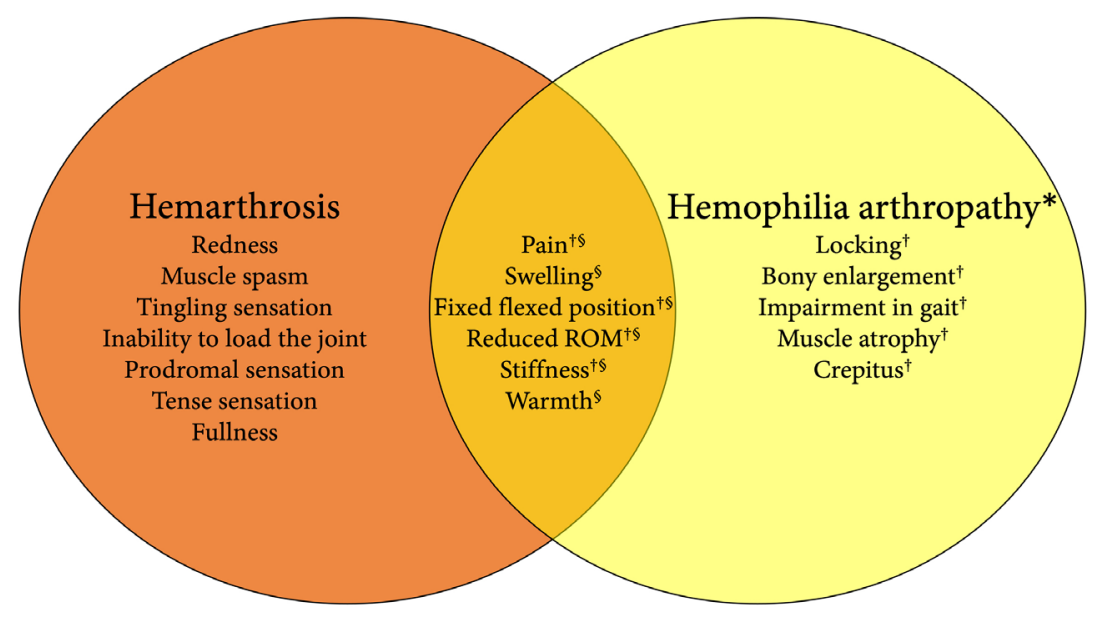

Figure 4. Overlap in symptoms between hemarthrosis and hemophilic arthropathy. ${ }^{\star}$ Including symptoms derived from literature on symptoms of osteoarthritis. ${ }^{\dagger}$ Symptoms associated with chronic complaints of hemophilic arthropathy. ${ }^{\S}$ Symptoms associated with flare-ups of hemophilic arthropathy. Adapted from Figure 2 of Timmer MA et al. Differentiating between signs of intra-articular joint bleeding and chronic arthropathy in haemophilia: a narrative review of the literature. Haemophilia 2015; 21: 289-96 [52]. (c) 2015 John Wiley \& Sons Ltd. 


\section{Adjusting the Physical Therapy Session for an Individual with Hemophilia}

\subsection{Taking a History}

A concise medical history describing the details of problematic symptoms and effectiveness of previous treatments is essential. The person's goals, motivations, and expectations of physical therapy should be discussed. Owing to the complexity of hemophilia management, musculoskeletal complications and other comorbidities, there are several questions that may help focus an individual's physical therapy needs, as shown in Table 3.

\subsection{Gaining the Trust of People with Hemophilia}

Most PWH are aware that their condition is rare and that few non-specialist community PTs have experience with it. By acknowledging this, the individual with hemophilia can be viewed as an expert on their condition and a potential resource for useful information. However, to gain the trust of and develop a relationship with an individual with hemophilia, questions that may highlight basic gaps in disease knowledge (e.g. asking how long they have had hemophilia) should be avoided. Individualized management is also important. Because PWH may have multiple joints affected by hemarthropathy, it is critical that the current functional issue is clearly identified and a tailored program is developed to address that area.

Table 3. Questions to ask PWH in a PT session.

Question to ask the individual
Rationale

- "Why have you come here today?"

PWH have many musculoskeletal problems, but may be compensating successfully for some of them. Just asking "What's wrong?" is therefore not useful, as PWH may be seeking help for a specific muscle, joint, or functional issue.

- "What is your current hemophilia treatment regimen?"

- "Are you on a prophylactic regimen, and if so are you following that plan?"

- "When was your last dose?"

- "Do you self-infuse?"

The PT should be familiar with the person's hemophilia treatment regimen and the basic principles of clotting factor treatment. Asking about these details will also reassure them that their PT understands hemophilia, and will highlight any problems with adherence to clotting factor treatment.

- "Who is your contact person at the HTC or hemophilia care provider?"
This may help to build a trusted relationship with an individual with hemophilia, and will provide the PT with a source of information on hemophilia. This question also ensures that the treating PT has direct communication with the person's treatment team, which is critical.
- "Do you have any other medical issues?"

- "Have you undergone surgery recently?"
As a result of previous medical treatment for hemophilia, some PWH may be co-infected with HIV or hepatitis $\mathrm{C}$, which has consequences for their treatment (e.g. side effects from HIV medications, increased osteoporosis risk). This may be a sensitive topic for many people, so should be approached with care.

The questions in this table were identified by the authors based on their experience in managing PWH. 


\subsection{Timing of Session}

A 20 - 30-minute physical therapy session may be too short for $\mathrm{PWH}$, especially if they have limited physical ability. As a result, a longer time slot is often required; for some, disrobing for examination may be difficult and time consuming, and managing the coordination for an exercise or transitioning on and off a treatment table may take longer. Physical therapy sessions should be coordinated and, if possible, scheduled to coincide with a person's regular factor infusion day (if they receive regular prophylactic infusions) to allow maximal hemostatic coverage and safety during the session. Individuals may need a space to infuse prior to the appointment. The HTC may advise PWH to alter their regular dosage or infuse more frequently while attending physical therapy.

Hematologists can be contacted if a special prescription to cover longer sessions is required. All individuals are different and should be asked for their preferred appointment time. For example, PWH with multiple arthrophic joints or individuals who have family commitments, and who need to manage multiple medical appointments, may not be able to attend early morning appointments easily.

\subsection{Practical Advice for Physical Therapy Sessions}

Apart from the points detailed above, there are several practical considerations to managing a PT session with an individual with hemophilia. "Green flags" are helpful best-practice actions that PTs can carry out for the benefit of PWH, and are listed in Table 4.

Likewise, it is essential to recognize "red flags", which are key indications that something is wrong or key actions that PTs should avoid. To prevent hemarthroses and recognize signs of bleeding, PTs should be aware of these red flags, the most important of which are listed in Table 5.

\section{Physical Therapy Exercises for People with Hemophilia}

Physical therapy helps to preserve function and slow mobility loss [5] [6]. Some PWH may have small arcs of available ROM, but they can still work on muscle strength. For PWH with fixed postures due to joint disease and muscle involvement, focusing on supportive strategies and prevention of further posture deformity is more important than corrective exercises. In $\mathrm{PWH}$, it is important to observe the entire lower extremity kinetic chain, as multiple joints may be affected.

Specific exercises may be recommended for physical therapy in $\mathrm{PWH}$ [41]:

- Balance exercises can help prevent falls (the risk of which increases with poor joint status and age) and may be suitable for PWH of all ages [73] [74].

- Isometric exercises are a safer alternative in PWH with damaged joints where movement and resistance may be harmful [41]. As a basic rule, $\mathrm{PWH}$ should progress from isometric to concentric strengthening exercises and from light to moderate resistance. 
Table 4. Green flags.

Green flag

When assessing musculoskeletal function and joint health, hemophilia-specific tools can be used.

3) When assessing pain in $\mathrm{PWH}$, consider

if there may be joint bleeding.

4) Include teaching of supportive postures while sitting and focus on joint protection in those with complications from established joint disease.

5) Encourage PWH to make creative use of their adaptive equipment and devices.

6) Work on functional strength (e.g. taking sweater on/off, putting on shoes, sit to stand, steps and stairs) in addition to classic muscle strengthening.

7) Isometric strengthening within a comfortable range may be considered the safest type of strengthening.

8) Day-to-day functional postures can be assessed in the clinical examination room.

9) Ensure PWH have sufficient hemostatic cover before commencing each physical therapy session, as recommended by their HTC.

10) It is essential to highlight the importance of adhering to the prescribed factor replacement regimen and the consequences of non-adherence (e.g. the consequences of bleeding).
Explanation

As progress can be slow for $\mathrm{PWH}$, thinking in longer timeframes can help (i.e. tracking progress at 2 - 4-week intervals rather than from day to day).

Tools such as the HJHS and the Hemophilia Activities List have been developed specifically to measure joint health and functional limitations in PWH, and have been validated [69]. These (and other) hemophilia-specific tools may be helpful to assess and improve outcomes, although PWH tend to prefer short tools [29] [70]. Hemophilia-specific tools should only be used by PTs trained in their use.

Differential diagnosis is difficult in hemophilia, as there are several pathologies with similar symptoms, and a hemophilic flare-up may also mask pain from a bleed. However, there are several characteristic signs and symptoms that indicate a bleed, including a prodromal sensation of aura, inability to load the joint, tingling sensation, lessening pain after clotting factor treatment, painful palpation of swelling, no locking joint and a feeling of fullness in the joint (see Figure 4) [51] [52].

Use of pillows, bolsters, and chairs can encourage a comfortable, ergonomic position and reduce stress on the joints, along with common joint protection techniques, equipment, and devices, as employed in the treatment of OA and rheumatoid arthritis.

PWH may use standard equipment and devices (e.g. a mobile walker), with or without modifications, in unconventional ways because they are unable to bear weight through upper extremity joints, owing to blood-induced joint damage. This approach helps PWH live their daily lives in the most effective way for them.

These functional strength exercises can be modified as needed (e.g. repetitions of bicep curls at a specific weight), at a level appropriate for the individual with hemophilia [41].

Isometrics focus solely on the muscle, without placing undue stress on joint structures, which may be damaged in PWH. Owing to the static nature of isometrics, it is commonly believed that there is little risk of impinging on hypertrophied synovial tissue, as could be the case during isotonic exercise. There should be a focus on a gradual progression from isometric to isotonic, within a pain-free ROM, and finally progressive, resistive exercises. Closed-chain versus open-chain formats should be considered to minimize risk.

This can be measured by examining how PWH conduct activities such as rising from a chair and standing at the sink to wash. Hints, tips, and prompts can be taught to enhance stability and control in such positions.

This treatment may or may not be necessary depending on the severity of hemophilia and the factor infusion regimen prescribed by the HTC. This may involve clotting factor treatment before each physical therapy session (for example in post-surgery cases), which most PWH will typically administer themselves.

This is particularly useful for groups in which adherence is often problematic, for example in adolescents or in older adults with poor venous access from years of infusing. 
Table 5. Red flags.

\begin{tabular}{ll}
\hline \multicolumn{1}{c}{ Red flag } & \multicolumn{1}{c}{ Explanation } \\
\hline & $\begin{array}{l}\text { When distinguishing between hip hemarthroses and iliopsoas bleeds, the former } \\
\text { cause pain with passive and active motion in any direction, and limited ROM; } \\
\text { 1) A combination of severe pain in the groin, } \\
\text { motor and sensory loss in femoral nerve }\end{array}$ \\
$\begin{array}{l}\text { the latter present with pain in the groin on active hip flexion or active and passive hip } \\
\text { (the "psoas sign") could indicate }\end{array}$ & $\begin{array}{l}\text { with a hemarthrosis or muscle bleed should be referred back to their HTC. However, } \\
\text { the psoas sign should be treated as a medical emergency, as psoas bleeds may be }\end{array}$ \\
an iliopsoas injury or bleed [18]. & life-threatening if not urgently treated with clotting factor replacement [18] [71].
\end{tabular}

2) Proprioceptive exercises may lead to bleeding if conducted too early after a hemarthrosis or in the presence of chronic synovitis.

Appropriate care should be taken.

3) Traditional posture exercises with end-range positions (such as aggressive stretching of fibrosed joints) should be avoided, as these exercises could aggravate arthropathy or generate new bleeds in joints or soft tissue.

4) PTs must stretch chronic joint flexion contractures with caution.

5) New or acute pain may indicate a new hemarthrosis or bleed.

6) PWH with inhibitors have a more complex form of hemophilia and their bleeding is more difficult to control; they should be managed only by PTs specialized in hemophilia at the HTC (where available).
These exercises are advanced, typically involving minor corrective movements and rotation in combination with weight bearing. They have a tendency to mechanically pinch a swollen, hypertrophied, highly vascular synovium, which can precipitate a hemarthrosis.

Hard end-feels and bony blocks can indicate arthropathy; in the presence of these signs, aggressive traditional exercises should be avoided. PWH may want to train on their own at home or at a fitness center without seeking guidance on appropriate muscle strength exercises from PTs. In these circumstances, it should be emphasized that end-range position exercises may cause hemarthroses and be detrimental to their joint health. The focus of physical therapy exercises should instead be on supportive strategies and within the available ROM in those with severe joint disease.

Stretching of any contracture (see Figure 5) in PWH should be undertaken with a high degree of caution and extremely slowly; acute contractures should be referred immediately to the HTC. This is very different from the approach used in the general population, where stretching is encouraged. Aggressive joint mobilization and high-velocity manipulations can cause a hemarthrosis and may be contraindicated.

Sometimes PWH struggle to distinguish bleed pain from arthropathy flares from soreness experienced at the beginning of a new exercise regimen.

PTs can assess the joint to determine the cause of pain before proceeding with treatment or exercise recommendations.

Inhibitors are a serious complication of hemophilia, where PWH develop antibodies against the clotting factor, and bleeding and joint disease can be more severe than usual [23] [24]. In these people, infusions of factor concentrates typically do not control bleeding, which may instead be managed with bypassing agents or an antibody therapeutic [7] [24]. Individuals with inhibitors often have more lengthy and poorly controlled coagulation, and more joint damage at younger ages vs those without inhibitors [23] [24] [72]. PTs treating these patients should proceed with great care, with delicate physical assessment and a slow progression of interventions. Ideally, PWH with inhibitors should be treated by experienced PTs in an HTC, where they may receive immediate assessment and intervention from a hematologist or nurse if needed.

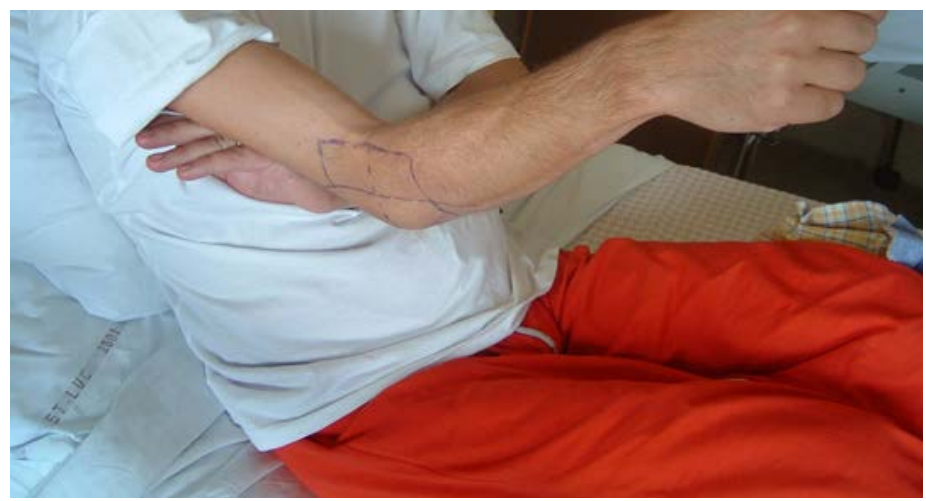

Figure 5. Elbow contracture in a man with hemophilia prior to elbow prosthesis. 
- Low-load static stretching/bracing or dynamic bracing, with a very gradual wearing/progression schedule. In general, active ROM should be preferred to passive ROM, as it is safer.

- Gentle myofascial release and other gentle manual techniques may be indicated for PWH [75] [76], in opposition to high-velocity manipulation and joint mobilization techniques.

Other exercises recommended for $\mathrm{PWH}$ can be found in a guide published by the World Federation of Hemophilia (WFH), or on the TalkingJoints ${ }^{\oplus}$ website [22] [41].

Starting exercise very gradually is advised, as many PWH may be sedentary or not participating in regular exercise. PTs should cautiously add to the regimen in the following weeks, progressively applying more advanced proprioceptive exercises, and taking into account the prolonged healing rate in PWH. PTs must remember that, as for anyone, prescribing too many exercises may cause adherence issues. This is especially true for $\mathrm{PWH}$ who could be managing multiple complications and may not be accustomed to physical activity. At all times, awareness of early signs of bleeding or synovitis, which may be precipitated by physical therapy or exercise, is essential, as is withholding exercise to an affected joint during a bleed.

For PWH who exhibit a risk of falling, as gait and functional mobility decline, the use of assistive devices and treatment techniques that focus on dynamic balance should be considered. PTs can suggest supportive or protective devices, such as orthoses, to improve function and reduce pain with minimal side effects [77]. Power mobility may be considered for PWH functioning at a community level of independence, to preserve and protect affected joints and reduce bleeding. In the authors' experience, a manual wheelchair may be more challenging, and individuals with affected upper extremity joints and frequent hemarthroses may be unable to propel them.

Even if the individual with hemophilia has advanced joint disease or poor joint health or function, the PT can still work towards and encourage a state of wellness with supervised exercise programs [39]. These should be part of the overall treatment and may help decrease hemarthroses and arthritic joint pain [7]. With guidance, communication and collaboration between the HTC, personal trainer and PT, PWH may be capable of undertaking an effective exercise program at a fitness facility.

\section{Communication with the Hemophilia Treatment Center and When to Refer to Hemophilia Specialists}

Communication between local PTs and the HTC is recommended throughout treatment as needed, starting from when the individual is referred. Effective communication with the HTC team and the specialist PT helps the local PT gain a wider knowledge of hemophilia. It can also help gain essential information, such as the needs and infusion schedules of PWH and any relevant regulations regarding prescriptions and reimbursement. The HTC can also answer any 
questions the PT may have, such as what to do if bleeding is suspected. It is important that the PT has a good understanding of the person's bleeding history and treatment regimen. PTs can also find helpful information on hemophilia from the WFH, National Hemophilia Foundation (NHF), and the European Association for Haemophilia and Allied Disorders (EAHAD), among others [78] [79] [80].

PTs should consider a referral back to the HTC if an individual with hemophilia:

- Thinks they have unusual or unfamiliar symptoms, uncontrolled bleeding, reduced response to factor treatment, or uncontrolled pain;

- Requires psychosocial support, displays mental health symptoms, or has problems with motivation/attending appointments.

\section{Conclusion}

PWH require individualized management while undergoing physical therapy. To improve their knowledge of the condition, PTs should acknowledge the expertise of PWH and communicate effectively with them and with their HTC to optimize care. In this article, the joint pathology in hemophilia and its consequences have been detailed, and practical advice on how to conduct physical therapy assessments and exercises have been presented. Therefore, this article is a useful practical resource for local PTs who are inexperienced in hemophilia. In conjunction with self-directed learning in haemophilia and in close communication with experienced HTCs, this article may help these PTs to learn more about the condition and the nuances of conducting physical therapy in PWH. With proper guidance and collaboration with the individual with hemophilia, HTC and experienced hemophilia PT, local PTs can treat PWH effectively and safely.

\section{Acknowledgements}

All authors contributed to the content of the manuscript, reviewed it critically at each stage of development and approved the final version for submission. We thank Flavia Sciota, Ph.D., AXON Communications, who received compensation from Novo Nordisk for medical writing and editorial assistance. Novo Nordisk was also provided with the opportunity to perform a medical accuracy review. This manuscript was developed in compliance with Good Publication Practice Guidelines.

\section{Conflicts of Interest}

A.F. has received consultancy honoraria from Aptevo, Blood CME Center, National Hemophilia Foundation, Novo Nordisk, and Shire, and reports no conflict of interest regarding this publication. G.B. has received honoraria from Novo Nordisk and Shire, funding to attend congresses from Pfizer, consultancy fees from Novo Nordisk, and personal fees from Shire. S.L. has received honoraria from Bayer, CSL-Behring, Novo Nordisk, Pfizer, Roche, Sobi, and Takeda. P.McL. has received honoraria from Novo Nordisk, Sobi and Takeda. 


\section{References}

[1] Pulles, A.E., Mastbergen, S.C., Schutgens, R.E., Lafeber, F.P. and van Vulpen, L.F. (2017) Pathophysiology of Hemophilic Arthropathy and Potential Targets for Therapy. Pharmacological Research, 115, 192-199. https://doi.org/10.1016/j.phrs.2016.11.032

[2] Mulder, K. and Llinás, A. (2004) The Target Joint. Haemophilia, 10(Suppl 4), 152-156. https://doi.org/10.1111/j.1365-2516.2004.00976.x

[3] Rodriguez-Merchan, E.C. (2016) Articular Bleeding in Hemophilia. Cardiovascular \& Hematological Disorders-Drug Targets, 16, 21-24. https://doi.org/10.2174/1871529X16666160613114506

[4] Blamey, G., Forsyth, A., Zourikian, N., Short, L., Jankovic, N., et al. (2010) Comprehensive Elements of a Physiotherapy Exercise Programme in Haemophilia-A Global Perspective. Haemophilia, 16(Suppl 5), 136-145. https://doi.org/10.1111/j.1365-2516.2010.02312.x

[5] Hanley, J., McKernan, A., Creagh, M.D., Classey, S., McLaughlin, P., et al. (2017) Guidelines for the Management of Acute Joint Bleeds and Chronic Synovitis in Haemophilia: A United Kingdom Haemophilia Centre Doctors' Organisation (UKHCDO) Guideline. Haemophilia, 23, 511-520. https://doi.org/10.1111/hae.13201

[6] Atilla, B. and Güney-Deniz, H. (2019) Musculoskeletal Treatment in Haemophilia. EFORT Open Reviews, 4, 230-239. https://doi.org/10.1302/2058-5241.4.180068

[7] Srivastava, A., Brewer, A.K., Mauser-Bunschoten, E.P., Key, N.S., Kitchen, S., et al. (2013) Guidelines for the Management of Hemophilia. Haemophilia, 19, e1-e47. https://doi.org/10.1111/j.1365-2516.2012.02909.x

[8] Scott, M.J., Xiang, H., Hart, D.P., Palmer, B., Collins, P.W., et al. (2019) Treatment Regimens and Outcomes in Severe and Moderate Haemophilia A in the UK: The THUNDER Study. Haemophilia, 25, 205-212. https://doi.org/10.1111/hae.13616

[9] Baker, J.R., Crudder, S.O., Riske, B., Bias, V. and Forsberg, A. (2005) A Model for a Regional System of Care to Promote the Health and Well-Being of People with Rare Chronic Genetic Disorders. American Journal of Public Health, 95, 1910-1916. https://doi.org/10.2105/AJPH.2004.051318

[10] Stephensen, D., de Kleijn, P., Matlary, R.E.D., Katzerova, M., McLaughlin, P., et al. (2019) Scope of Practice of Haemophilia Physiotherapists: A European Survey. Haemophilia, 25, 514-520. https://doi.org/10.1111/hae.13727

[11] Jansen, N.W.D., Roosendaal, G. and Lafeber, F.P.J.G. (2008) Understanding Haemophilic Arthropathy: An Exploration of Current Open Issues. British Journal of Haematology, 143, 632-640. https://doi.org/10.1111/j.1365-2141.2008.07386.x

[12] Rodriguez-Merchan, E.C. (2008) Articular Bleeding (Hemarthrosis) in Hemophilia. World Federation of Hemophilia. https://elearning.wfh.org/resource/articular-bleeding-hemarthrosis-in-hemophilia-a n-orthopedists-point-of-view/

[13] Kuijlaars, I.A.R., Timmer, M.A., de Kleijn, P., Pisters, M.F. and Fischer, K. (2017) Monitoring Joint Health in Haemophilia: Factors Associated with Deterioration. Haemophilia, 23, 934-940. https://doi.org/10.1111/hae.13327

[14] Lobet, S., Hermans, C. and Lambert, C. (2014) Optimal Management of Hemophilic Arthropathy and Hematomas. Journal of Blood Medicine, 5, 207-218. https://doi.org/10.2147/JBM.S50644

[15] Manco-Johnson, M.J., Abshire, T.C., Shapiro, A.D., Riske, B., Hacker, M.R., et al. 
(2007) Prophylaxis versus Episodic Treatment to Prevent Joint Disease in Boys with Severe Hemophilia. New England Journal of Medicine, 357, 535-544. https://doi.org/10.1056/NEJMoa067659

[16] Goto, M., Takedani, H., Nitta, O. and Kawama, K. (2015) Joint Function and Arthropathy Severity in Patients with Hemophilia. Journal of the Japanese Physical Therapy Association, 18, 15-22. https://doi.org/10.1298/jjpta.Vol18 003

[17] Philipp, C. (2010) The Aging Patient with Hemophilia: Complications, Comorbidities, and Management Issues. Hematology/ American Society of Hematology Education Program, 2010, 191-196. https://doi.org/10.1182/asheducation-2010.1.191

[18] Buzzard, B. and Jones, P. (1988) Physiotherapy Management of Haemophilia: An Update. Physiotherapy, 74, 221-226. https://doi.org/10.1016/S0031-9406(10)63535-3

[19] Escobar, M.A., Brewer, A., Caviglia, H., Forsyth, A., Jimenez-Yuste, V., et al. (2018) Recommendations on Multidisciplinary Management of Elective Surgery in People with Haemophilia. Haemophilia, 24, 693-702. https://doi.org/10.1111/hae.13549

[20] De la Corte-Rodriguez, H. and Rodriguez-Merchan, E.C. (2013) The Role of Physical Medicine and Rehabilitation in Haemophiliac Patients. Blood Coagulation \& Fibrinolysis, 24, 1-9. https://doi.org/10.1097/MBC.0b013e32835a72f3

[21] Guodemar-Pérez, J., Ruiz-López, M., Rodríguez-López, E., García-Fernández, P. and Hervás-Pérez, J.P. (2017) Physiotherapy Treatments in Musculoskeletal Pathologies Associated with Haemophilia. A Literature Review. Hamostaseologie, 38, 141-149.

[22] Novo Nordisk (2016) TalkingJoints ${ }^{\oplus}$. http://www.novonordisk.com/about-novo-nordisk/changing-haemophilia/changing -haemophilia-focus-areas/joint-outcomes/talking-joints.html

[23] Morfini, M., Haya, S., Tagariello, G., Pollmann, H., Quintana, M., et al. (2007) European Study on Orthopaedic Status of Haemophilia Patients with Inhibitors. Haemophilia, 13, 606-612. https://doi.org/10.1111/j.1365-2516.2007.01518.x

[24] Ljung, R., Auerswald, G., Benson, G., Dolan, G., Duffy, A., et al. (2019) Inhibitors in Haemophilia A and B: Management of Bleeds, Inhibitor Eradication and Strategies for Difficult-to-Treat Patients. European Journal of Haematology, 102, 111-122. https://doi.org/10.1111/ejh.13193

[25] Bolton-Maggs, P.H. and Pasi, K.J. (2003) Haemophilias A and B. The Lancet, 361, 1801-1809. https://doi.org/10.1016/S0140-6736(03)13405-8

[26] Riley, R.R., Witkop, M., Hellman, E. and Akins, S. (2011) Assessment and Management of Pain in Haemophilia Patients. Haemophilia, 17, 839-845. https://doi.org/10.1111/j.1365-2516.2011.02567.x

[27] Tagliaferri, A., Franchini, M., Rivolta, G.F., Farace, S., Quintavalle, G., et al. (2018) Pain Assessment and Management in Haemophilia: A Survey among Italian Patients and Specialist Physicians. Haemophilia, 24, 766-773. https://doi.org/10.1111/hae.13600

[28] Humphries, T.J. and Kessler, C.M. (2015) Managing Chronic Pain in Adults with Haemophilia: Current Status and Call to Action. Haemophilia, 21, 41-51. https://doi.org/10.1111/hae.12526

[29] Bradshaw, E., McClellan, C., Whybrow, P. and Cramp, F. (2019) Physiotherapy Outcome Measures of Haemophilia Acute Bleed Episodes: What Matters to Patients? Haemophilia, 25, 1066-1072. https://doi.org/10.1111/hae.13840

[30] Hawker, G.A., Mian, S., Kendzerska, T. and French, M. (2011) Measures of Adult 
Pain: Visual Analog Scale for Pain (VAS Pain), Numeric Rating Scale for Pain (NRS Pain), McGill Pain Questionnaire (MPQ), Short-Form McGill Pain Questionnaire (SF-MPQ), Chronic Pain Grade Scale (CPGS), Short Form-36 Bodily Pain Scale (SF-36 BPS), and Measure of Intermittent and Constant Osteoarthritis Pain (ICOAP). Arthritis Care \& Research, 63(Suppl 11), S240-S252. https://doi.org/10.1002/acr.20543

[31] Herdman, M., Gudex, C., Lloyd, A., Janssen, M., Kind, P., et al. (2011) Development and Preliminary Testing of the New Five-Level Version of EQ-5D (EQ-5D-5L). Quality of Life Research, 20, 1727-1736. https://doi.org/10.1007/s11136-011-9903-x

[32] Torres-Ortuño, A., Cuesta-Barriuso, R., Nieto-Munuera, J., Galindo-Piñana, P. and López-Pina, J.A. (2019) Coping Strategies in Young and Adult Haemophilia Patients: A Tool for the Adaptation to the Disease. Haemophilia, 25, 392-397. https://doi.org/10.1111/hae.13743

[33] Wang, C.Z., Moss, J. and Yuan, C.S. (2015) Commonly Used Dietary Supplements on Coagulation Function during Surgery. Medicines (Basel), 2, 157-185. https://doi.org/10.3390/medicines2030157

[34] Franchini, M., Tagliaferri, A. and Mannucci, P.M. (2007) The Management of Hemophilia in Elderly Patients. Clinical Interventions in Aging, 2, 361-368.

[35] Lobet, S., Pendeville, E., Dalzell, R., Defalque, A., Lambert, C., et al. (2008) The Role of Physiotherapy after Total Knee Arthroplasty in Patients with Haemophilia. Haemophilia, 14, 989-998. https://doi.org/10.1111/j.1365-2516.2008.01748.x

[36] Souza, F.M., McLaughlin, P., Pereira, R.P., Minuque, N.P., Mello, M.H., et al. (2013) The Effects of Repetitive Haemarthrosis on Postural Balance in Children with Haemophilia. Haemophilia, 19, e212-e217. https://doi.org/10.1111/hae.12106

[37] Ragni, M.V. (2011) Aging in Haemophilia: Getting to the Heart of the Matter. Thrombosis and Haemostasis, 105, 207-208. https://doi.org/10.1160/TH10-12-0818

[38] Fearn, M., Hill, K., Williams, S., Mudge, L., Walsh, C., et al. (2010) Balance Dysfunction in Adults with Haemophilia. Haemophilia, 16, 606-614. https://doi.org/10.1111/j.1365-2516.2010.02200.x

[39] Mulvany, R., Zucker-Levin, A.R., Jeng, M., Joyce, C., Tuller, J., et al. (2010) Effects of a 6-Week, Individualized, Supervised Exercise Program for People with Bleeding Disorders and Hemophilic Arthritis. Physical Therapy, 90, 509-526. https://doi.org/10.2522/ptj.20080202

[40] Brown, D., Claffey, A. and Harding, R. (2016) Evaluation of a Physiotherapy-Led Group Rehabilitation Intervention for Adults Living with HIV: Referrals, Adherence and Outcomes. AIDS Care, 28, 1495-1505. https://doi.org/10.1080/09540121.2016.1191611

[41] Mulder, K. (2006) Exercises for People with Hemophilia. World Federation of Hemophilia. http://elearning.wfh.org/resource/exercises-for-people-with-hemophilia/

[42] Lobet, S., Detrembleur, C., Francq, B. and Hermans, C. (2010) Natural Progression of Blood-Induced Joint Damage in Patients with Haemophilia: Clinical Relevance and Reproducibility of Three-Dimensional Gait Analysis. Haemophilia, 16, 813-821. https://doi.org/10.1111/j.1365-2516.2010.02245.x

[43] Lobet, S., Detrembleur, C. and Hermans, C. (2013) Impact of Multiple Joint Impairments on the Energetics and Mechanics of Walking in Patients with Haemophilia. Haemophilia, 19, e66-e72. https://doi.org/10.1111/hae.12001

[44] Lobet, S., Detrembleur, C., Massaad, F. and Hermans, C. (2013) Three-Dimensional Gait Analysis Can Shed New Light on Walking in Patients with Haemophilia. The 
Scientific World Journal, 2013, Article ID: 284358. https://doi.org/10.1155/2013/284358

[45] Lobet, S., McCarthy, A., Hermans, C., Peerlinck, K., Matricali, G.A., et al. (2017) Biomechanical Markers and Theoretical Concepts Related to Haemophilic Ankle and Subtalar Joint Arthropathy: Introducing the Term "Haemophilic Tarsal PanArthropathy”. Haemophilia, 23, e250-e258. https://doi.org/10.1111/hae.13202

[46] Wyseure, T., Mosnier, L.O. and von Drygalski, A. (2016) Advances and Challenges in Hemophilic Arthropathy. Seminars in Hematology, 53, 10-19. https://doi.org/10.1053/j.seminhematol.2015.10.005

[47] Vanhoenacker, F., Van Dyck, P., Gielen, J. and De Schepper, A. (2014) Tumor and Tumor-Like Lesions of Soft Tissue. In: Davies, A., Grainger, A. and James, S., Eds., Imaging of the Hand and Wrist. Techniques and Applications, Springer Science \& Business Media, Berlin, Heidelberg, 317-348. https://doi.org/10.1007/174 2010113

[48] Falah, M., Nierenberg, G., Soudry, M., Hayden, M. and Volpin, G. (2010) Treatment of Articular Cartilage Lesions of the Knee. International Orthopaedics, 34, 621-630. https://doi.org/10.1007/s00264-010-0959-y

[49] Hung, Y.J. (2015) Neuromuscular Control and Rehabilitation of the Unstable Ankle. World Journal of Orthopedics, 6, 434-438. https://doi.org/10.5312/wjo.v6.i5.434

[50] Ceponis, A., Wong-Sefidan, I., Glass, C.S. and von Drygalski, A. (2013) Rapid Musculoskeletal Ultrasound for Painful Episodes in Adult Haemophilia Patients. Haemophilia, 19, 790-798. https://doi.org/10.1111/hae.12175

[51] Timmer, M.A., Pisters, M.F., de Kleijn, P., Veenhof, C., Laros-van Gorkom, B.A., et al. (2016) How Do Patients and Professionals Differentiate between Intra-Articular Joint Bleeds and Acute Flare-Ups of Arthropathy in Patients with Haemophilia? Haemophilia, 22, 368-373. https://doi.org/10.1111/hae.12858

[52] Timmer, M.A., Pisters, M.F., de Kleijn, P., de Bie, R.A., Fischer, K., et al. (2015) Differentiating between Signs of Intra-Articular Joint Bleeding and Chronic Arthropathy in Haemophilia: A Narrative Review of the Literature. Haemophilia, 21, 289-296. https://doi.org/10.1111/hae.12667

[53] Stephensen, D., Bladen, M. and McLaughlin, P. (2018) Recent Advances in Musculoskeletal Physiotherapy for Haemophilia. Therapeutic Advances in Hematology, 9, 227-237. https://doi.org/10.1177/2040620718784834

[54] Melchiorre, D., Manetti, M. and Matucci-Cerinic, M. (2017) Pathophysiology of Hemophilic Arthropathy. Journal of Clinical Medicine, 6, E63.

https://doi.org/10.3390/jcm6070063

[55] Kulkarni, R. and Lusher, J. (2001) Perinatal Management of Newborns with Haemophilia. British Journal of Haematology, 112, 264-274. https://doi.org/10.1046/j.1365-2141.2001.02362.x

[56] Krasnokutsky, S., Attur, M., Palmer, G., Samuels, J. and Abramson, S.B. (2008) Current Concepts in the Pathogenesis of Osteoarthritis. Osteoarthritis and Cartilage, 16(Suppl 3), S1-S3. https://doi.org/10.1016/j.joca.2008.06.025

[57] Blagojevic, M., Jinks, C., Jeffery, A. and Jordan, K.P. (2010) Risk Factors for Onset of Osteoarthritis of the Knee in Older Adults: A Systematic Review and Meta-Analysis. Osteoarthritis and Cartilage, 18, 24-33. https://doi.org/10.1016/j.joca.2009.08.010

[58] Shane Anderson, A. and Loeser, R.F. (2010) Why Is Osteoarthritis an Age-Related Disease? Best Practice \& Research Clinical Rheumatology, 24, 15-26. https://doi.org/10.1016/j.berh.2009.08.006 
[59] Pease, C.T., Bhakta, B.B., Devlin, J. and Emery, P. (1999) Does the Age of Onset of Rheumatoid Arthritis Influence Phenotype?: A Prospective Study of Outcome and Prognostic Factors. Rheumatology (Oxford), 38, 228-234. https://doi.org/10.1093/rheumatology/38.3.228

[60] Choy, E. (2012) Understanding the Dynamics: Pathways Involved in the Pathogenesis of Rheumatoid Arthritis. Rheumatology (Oxford), 51(Suppl 5), v3-v11. https://doi.org/10.1093/rheumatology/kes113

[61] Smolen, J.S., Aletaha, D. and McInnes, I.B. (2016) Rheumatoid Arthritis. The Lancet, 388, 2023-2038. https://doi.org/10.1016/S0140-6736(16)30173-8

[62] Saag, K.G., Cerhan, J.R., Kolluri, S., Ohashi, K., Hunninghake, G.W., et al. (1997) Cigarette Smoking and Rheumatoid Arthritis Severity. Annals of the Rheumatic Diseases, 56, 463-469. https://doi.org/10.1136/ard.56.8.463

[63] Solomon, D.H., Kremer, J., Curtis, J.R., Hochberg, M.C., Reed, G., et al. (2010) Explaining the Cardiovascular Risk Associated with Rheumatoid Arthritis: Traditional Risk Factors versus Markers of Rheumatoid Arthritis Severity. Annals of the Rheumatic Diseases, 69, 1920-1925. https://doi.org/10.1136/ard.2009.122226

[64] Centers for Disease Control and Prevention (2010) Normal Joint Range of Motion Study. https://www.cdc.gov/ncbddd/jointrom/

[65] Soucie, J.M., Cianfrini, C., Janco, R.L., Kulkarni, R., Hambleton, J., et al. (2004) Joint Range-of-Motion Limitations among Young Males with Hemophilia: Prevalence and Risk Factors. Blood, 103, 2467-2473.

[66] Soucie, J.M., Wang, C., Forsyth, A., Funk, S., Denny, M., et al. (2011) Range of Motion Measurements: Reference Values and a Database for Comparison Studies. Haemophilia, 17, 500-507. https://doi.org/10.1111/j.1365-2516.2010.02399.x

[67] Gilbert, M.S. (2000) Musculoskeletal Complications of Haemophilia: The Joint. Haemophilia, 6(Suppl 1), 34-37. https://doi.org/10.1046/j.1365-2516.2000.00044.x

[68] Blanchette, V.S., Key, N.S., Ljung, L.R., Manco-Johnson, M.J., van den Berg, H.M., et al. (2014) Definitions in Hemophilia: Communication from the SSC of the ISTH. Journal of Thrombosis and Haemostasis, 12, 1935-1939.

https://doi.org/10.1111/jth.12672

[69] McLaughlin, P., Morris, R. and Chowdary, P. (2018) Investigating the Relationship between the HJHS and HAL in Routine Clinical Practice: A Retrospective Review. Haemophilia, 24, 988-994. https://doi.org/10.1111/hae.13614

[70] Seuser, A., Djambas Khayat, C., Negrier, C., Sabbour, A. and Heijnen, L. (2018) Evaluation of Early Musculoskeletal Disease in Patients with Haemophilia: Results from an Expert Consensus. Blood Coagulation \& Fibrinolysis, 29, 509-520. https://doi.org/10.1097/MBC.0000000000000767

[71] Goodfellow, J., Fearn, C.B. and Matthews, J.M. (1967) Iliacus Haematoma. A Common Complication of Haemophilia. The Journal of Bone and Joint Surgery, 49, 748-756. https://doi.org/10.1302/0301-620X.49B4.748

[72] Gomez, K., Klamroth, R., Mahlangu, J., Mancuso, M.E., Mingot, M.E., et al. (2014) Key Issues in Inhibitor Management in Patients with Haemophilia. Blood Transfusion, 12(Suppl 1), s319-s329. https://doi.org/10.2450/2013.0246-12

[73] Negrier, C., Seuser, A., Forsyth, A., Lobet, S., Llinas, A., et al. (2013) The Benefits of Exercise for Patients with Haemophilia and Recommendations for Safe and Effective Physical Activity. Haemophilia, 19, 487-498. https://doi.org/10.1111/hae.12118

[74] Lobet, S., Lambert, C. and Hermans, C. (2016) Stop Only Advising Physical Activity in Adults with Haemophilia... Prescribe It Now! The Role of Exercise Therapy and 
Nutrition in Chronic Musculoskeletal Diseases. Haemophilia, 22, e554-e556. https://doi.org/10.1111/hae.13073

[75] Donoso-Úbeda, E., Meroño-Gallut, J., López-Pina, J.A. and Cuesta-Barriuso, R. (2018) Safety of Fascial Therapy in Adult Patients with Hemophilic Arthropathy of Ankle. A Cohort Study. Musculoskeletal Science and Practice, 35, 90-94. https://doi.org/10.1016/j.msksp.2018.03.003

[76] Donoso-Úbeda, E., Meroño-Gallut, J., López-Pina, J.A. and Cuesta-Barriuso, R. (2019) Effect of Manual Therapy in Patients with Hemophilia and Ankle Arthropathy: A Randomized Clinical Trial. Clinical Rehabilitation, 34, 111-119. https://doi.org/10.1177/0269215519879212

[77] Lobet, S., Detrembleur, C., Lantin, A.C., Haenecour, L. and Hermans, C. (2012) Functional Impact of Custom-Made Foot Orthoses in Patients with Haemophilic Ankle Arthropathy. Haemophilia, 18, e227-e235. https://doi.org/10.1111/j.1365-2516.2011.02711.x

[78] World Federation of Hemophilia. https://www.wfh.org/en/page.aspx?pid=492

[79] National Hemophilia Foundation. https://www.hemophilia.org/

[80] European Association for Haemophilia and Allied Disorders. http://eahad.org/ 